\title{
Localisation des gènes MPI, PKM2, NP sur le chromosome 3 du porc (Sus scrofa L.) et analyse cytogénétique d'une lignée de hamster chinois issue de la DON (wg3h)
}

\author{
Geneviève ECHARD, J. GELLIN et M. GILLOIS \\ avec la collaboration technique de Chantal de BANIZETTE \\ I.N.R.A., Laboratoire de Génétique cellulaire \\ Centre de Recherches de Toulouse, B.P. 12, F 31320 Castanet-Tolosan
}

\begin{abstract}
Résumé
L'analyse par électrophorèse de clones cellulaires hybrides interspécifiques porc-hamster chinois nous a permis de démontrer que les gènes MPI, PKM2, NP sont synténiques chez le porc (GELLIN et al., 1981). Le sous-clonage d'un de ces hybrides, l'analyse de l'expression de ces gènes dans les sous-clones et l'analyse simultanée de leur caryotype permet de localiser MPI, PKM2, NP sur le chromosome 3 du porc. Cette étude nécessite une connaissance précise du caryotype de la lignée permanente de hamster chinois utilisée (wg3h).
\end{abstract}

Mots clés : Carte génique, synténie, assignation, chromosome, caryotype.

\section{Summary}

Assignment of the genes MPI, PKM2, NP to pig chromosome 3 and karyotype analysis of the chinese hamster line wghh (derived from the DON strain)

The MPI, PKM2 and NP genes were shown to be syntenic in pigs after electrophoretic analysis of interspecific pig chinese hamster somatic cell hybrids (GELLIN et al., 1981). The expression of these genes was studied, together with karyotyping in subclones of one such parental hybrid clone derived from the permanent Chinese (hamster line wg3). Since the karyotype of this line is well understood, we were able to assign the genes MPI, PKM2 and NP to pig chromosome 3.

Key words: Gene mapping, synteny, assignment, chromosome, karyotype.

\section{Introduction}

La connaissance de la carte génique du porc est peu avancée. La première liaison génétique a été observée en 1964 par ANDRESEN \& BAKER entre les locus des groupes sanguins C et J. En 1976, HRUBAN et al. démontrent que ces derniers sont liés au 
complexe d'histocompatibilité SLA. Quatre autres groupes de liaison portés par les autosomes ont depuis été observés et sont décrits en détail par Ollivier \& Sellier (1982). Enfin 2 maladies : le «Splay leg » et le "Tremor AIII », sont attribuées au chromosome $\mathrm{X}$. Le développement des méthodes d'hybridation somatique interspécifique a permis de progresser dans la connaissance des cartes géniques. En ce qui concerne le porc, 4 résultats ont été acquis par ces méthodes :

- les gènes HPRT, G6PD, PGK et GLA sont synténiques comme chez les autres mammifères et portés par le chromosome X (GELLIN et al., 1980 ; FÖRSTER et al., 1980 ; LEONG et al., 1982);

- les gènes MPI, PKM2, NP sont synténiques (GELlin et al., 1981) ;

- le gène SOD1 est localisé sur le chromosome 9 (LEONG et al., 1982) ;

- les gènes LDHB, TPI, PEPB ne sont pas liés chez le porc contrairement à ce qui s'observe chez l'homme et d'autres mammifères (GELlin et al., 1979).

Nous présentons ici l'analyse cytogénétique de sous-clones obtenus à partir d'un clone hybride, porc hamster chinois, positif pour la synténie MPI, PKM2, NP, qui montre sa localisation sur le chromosome 3 . Cette analyse nous amène à décrire le caryotype de la lignée de hamster chinois wg3h.

\section{Matériel et méthodes}

\section{A. Lignées cellulaires hybridés et conditions de culture}

Les méthodes d'hybridation et de sélection de clones hybrides ont été décrites par ailleurs (GELLIN et al., 1980). Un clone hybride a été choisi pour son expression positive des 3 enzymes MPI, PKM2, NP : le clone PFchcl ${ }_{2}$ A (GEllin et al., 1981), plus simplement appelé PFA. Ce clone a été obtenu après fusion de fibroblastes d'une truie avec la souche permanente de hamster chinois $\mathrm{wg} 3 \mathrm{~h} \mathrm{cl}_{2}$.

Le sous-clonage est effectué de la façon suivante : 40 bouteilles de $60 \mathrm{ml}$ sont ensemencées à raison de 10 cellules par bouteille. Au bout de 10 à $15 \mathrm{j}$, de 0 à 4 colonies apparaissent par bouteille. Une seule d'entre elles est prélevée et transférée dans une bouteille propre. Les sous-clones sont cultivés comme les clones dans du milieu RPMI 1640 contenant du HAT et 10 p. 100 de sérum de veau nouveau-né.

\section{B. Analyse enzymatique}

Les électrophorèses sont réalisées selon les techniques déjà décrites (GELLIN et al., 1981). La récolte des cellules est faite en même temps et au même passage pour l'analyse enzymatique et les caryotypes.

Les enzymes suivantes ont été analysées dans les sous-clones :

- G6PD (EC no 1.1.1.49) Glucose-6-phosphate déshydrogénase ;

- GLA (EC no 3.2.1.22) $\alpha$-Galactosidase ;

- MPI (EC no 5.3.1.8.) Mannose phosphate isomérase ;

- PKM2 (EC no 2.7.1.40) Pyruvate kinase 2 ; 
- NP (EC no 2.4.2.1.) Nucléoside phosphorylase ;

- AK1 (EC no 2.7.4.3.) Adénylate kinase 1.

Les symboles utilisés pour nommer les enzymes et leurs gènes respectifs sont ceux proposés par le Comité International de Nomenclature (International System for Human Gene Nomenclature 1979).

\section{Analyse cytogénétique}

Pour chaque sous-clone, le caryotype est réalisé de la façon suivante : $2,5 \times 10^{6}$ cellules sont ensemencées dans un flacon de verre de $125 \mathrm{ml}$. Après $48 \mathrm{~h}$ de culture, lorsque la population est en phase de croissance logarithmique, les cellules sont bloquées en métaphase par la colcémide $(0,05 \mu \mathrm{g} / \mathrm{ml})$ pendant 2 ou $3 \mathrm{~h}$. Les cellules en mitose sont décollées du tapis cellulaire par agitation. Après une centrifugation de $30 \mathrm{~s}$ à 1500 tours $/ \mathrm{mn}$, le culot est remis en suspension dans $1 \mathrm{ml}$ de milieu hypotonique $(\mathrm{KCl} 0,7 \mathrm{p} .100)$ pendant $3 \mathrm{mn}$ à $37^{\circ} \mathrm{C}$. Une $2^{\mathrm{e}}$ centrifugation permet d'éliminer 80 p. $100 \mathrm{du}$ milieu hypotonique, le culot est remis en suspension dans les 20 p. 100 restant. Les cellules sont ensuite tuées et fixées à l'aide d'un mélange méthanoVacide acétique $(3 \mathrm{~V} / 1 \mathrm{~V})$. Une $3^{\mathrm{e}}$ centrifugation permet de remettre les métaphases dans du fixateur pur. Les chromosomes sont étalés sur lame selon la technique classique. Les bandes G.T.G. (Bandes G, Trypsine, Giemsa) sont obtenues en utilisant la technique de WANG \& FEDEROFF (1972) modifiée par FrANCKe \& OLIVER (1978). Le caryotype est réalisé en classant d'abord les chromosomes de la lignée $\mathrm{wg} \mathrm{h} \mathrm{cl}_{2}$ et ensuite les chromosomes de porc. Nous avons adopté pour le hamster chinois (Cricetulus griseus) la nomenclature standardisée proposée par RAY \& MOHANDAS (1976) et pour le porc, l'ordre proposé dans les « Proceedings of the First International Conference for the Standardisation of Banded Karyotypes of Domestic Animals » (READING, 1976). 50 mitoses sont analysées pour chaque clone.

\section{Résultats}

\section{A. Obtention de sous-clones et analyse enzymatique}

Trente-trois sous-clones ont été isolés à partir de l'hybride PFA, 28 d'entre eux ont poussé normalement. Chaque sous-clone a été analysé pour l'expression de MPI, PKM2, NP. La ségrégation de ces enzymes confirme la synténie : ces 3 enzymes sont gardées ensemble dans 15 sous-clones et perdues ensemble dans 11 sous-clones, 2 sont discordants MPI, PKM2 négatifs et NP positifs. Parmi ces 28 sous-clones, 14 ont été sélectionnés pour l'analyse caryologique, 7 nettement positifs pour MPI, PKM2, NP et 7 nettement négatifs. Pour ces 14 sous-clones retenus une analyse enzymatique a été faite au même passage que l'analyse cytogénétique.

\section{B. Analyse cytogénétique}

1. Caryotype de la souche permanente wg3h et du clone qui en dérive : wg3h $\mathrm{cl}_{2}$

La lignée wg3h est issue de la lignée DON établie en 1962 par HSU à partir d'un poumon de hamster chinois mâle. En 1971, WESTERVELD et al., font dériver 3 clones 
de la lignée .DON. L'un d'entre eux est appelé wg3h, il est HPRT négatif et diploïde (22 chromosomes). Aucune anomalie chromosomique n'a été décrite à cette époque. Le caryotype actuel de la wg3h comporte de nombreux chromosomes remaniés par rapport à celui du hamster chinois. Il existe une moyenne de 22 chromosomes (entre 20 et 23), aucun centromère n'est perdu mais on observe 7 chromosomes anormaux (fig. 1). Le sous-clone $\mathrm{wg} 3 \mathrm{~h} \mathrm{cl}_{2}$ utilisé pour la fabrication des hybrides n'a que 21 chromosomes en moyenne, l'un des chromosomes 10 étant perdu.

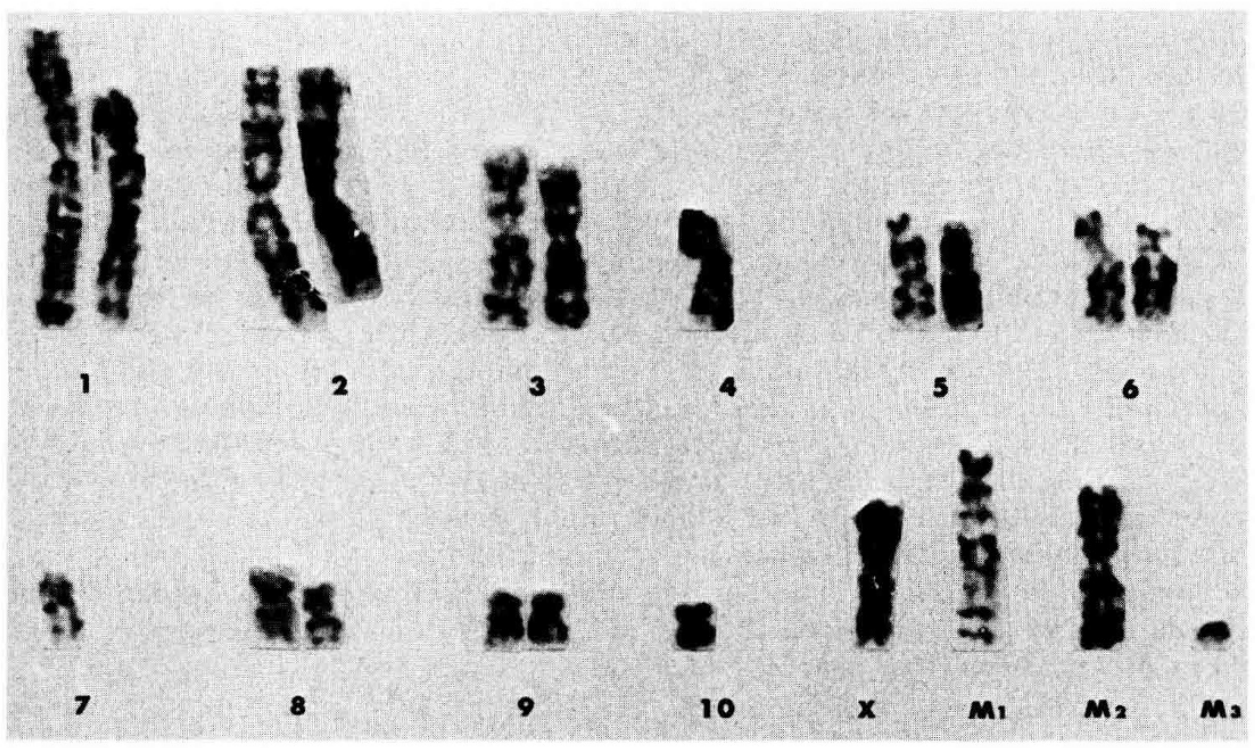

FIG. 1

Caryotype du clone wg3h $\mathrm{cl}_{2}$.

Clone wg3h $\mathrm{cl}_{2}$ karyotype.

Les paires 3,8,9 sont diploïdes. Les paires $4,7,10$ sont haploïdes. Il existe 3 chromosomes délétés : $1(1 \mathrm{p} 19) ; 2(2 \mathrm{q} 23) ; 5\left(\mathrm{p}^{-}\right)$et 4 chromosomes anormaux : $6\left(\mathrm{q}^{+}\right) ; \mathrm{M} 1, \mathrm{M} 2, \mathrm{M} 3$. Le chromosome $\mathrm{Y}$ est perdu ou fait partie d'un chromosome rcmanié. Comme dans les souches anciennes un lot haploïde de chromosomes est resté normal (ECHARD, 1974.).

Pairs 3,8,9 are diploid. Pairs 4,7,10 are haploid. There are 3 deleted chromosomes : 1 (1p19) ; $2(2 q 23) ; 5\left(p^{-}\right)$and 4 abnormal chromosomes : $6\left(q^{+}\right) ; M 1, M 2$, M3. The Y chromosome is lost or is part of a rearranged chromosome. As in ancient strains, and haploid set of chromosomes remained unchanged (ECHARD, 1974).

\section{Analyse cytogénétique du clone parental PFA et des sous-clones}

Le clone hybride PFA comporte un nombre moyen de 29 chromosomes. Le génome provenant de $\mathrm{wg} 3 \mathrm{~h} \mathrm{cl}_{2}$ est homogène avec 19 chromosomes : un chromosome 9 est perdu ainsi que le marqueur M3 (fig. 2). Le génome provenant du porc comprend 10 chromosomes : 7 sont normaux (les $\mathrm{n}^{\text {s }} 1,3,6,7,8,11, \mathrm{X}$ ) et 3 sont remaniés, l'un est identifiable $\left(16 p^{+}\right)$, les deux autres ne le sont pas : (R1 et $\left.R 2\right)$, mais ségregent dans 


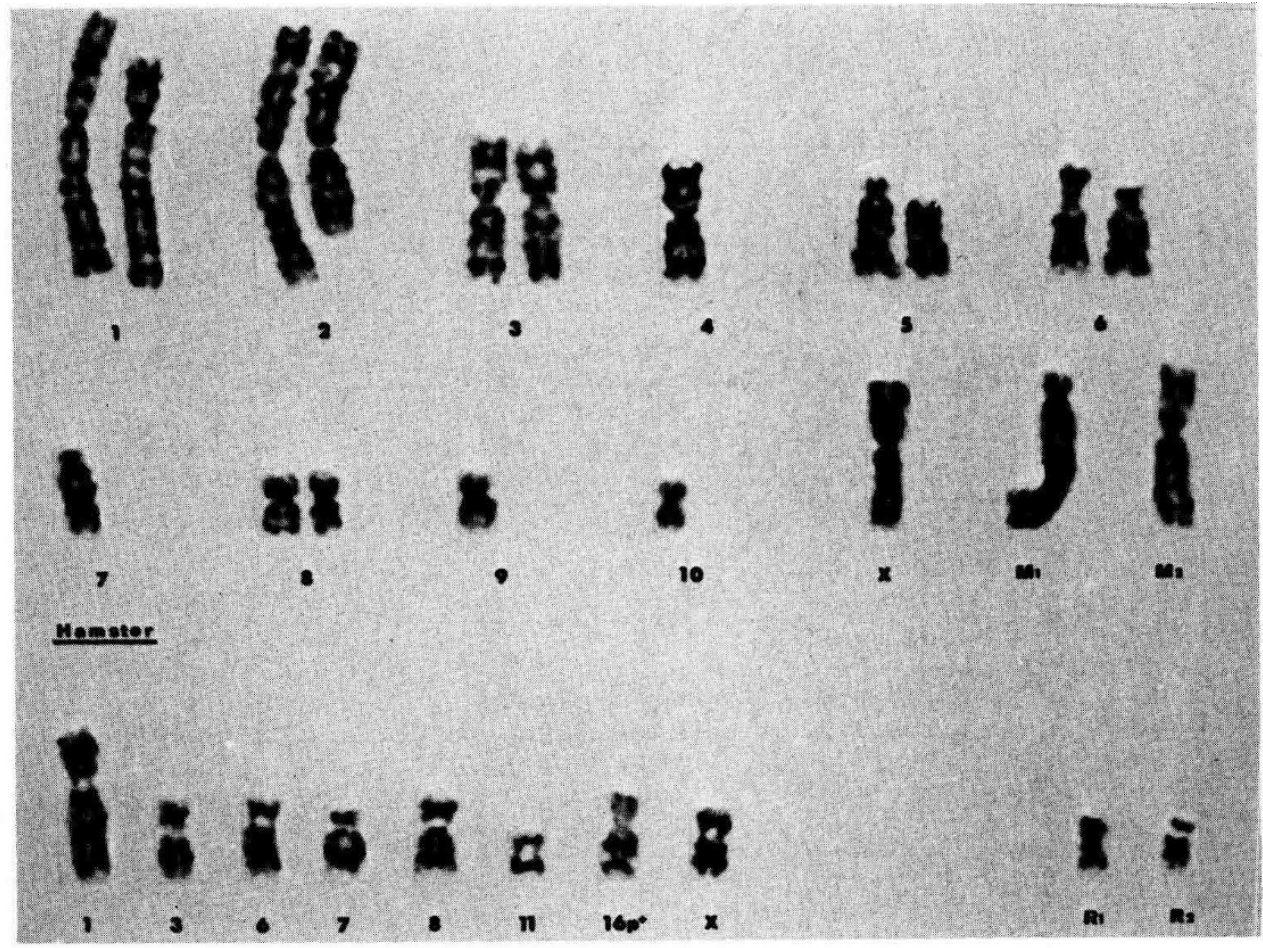

Fig. 2

Caryotype du clone hybride parental PFA.

Parental hybrid clone PFA karyotype

les sous-clones comme des chromosomes de porc (tabl. 1). Tous les sous-clones contiennent les chromosomes du hamster présents dans le clone PFA. La répartition des chromosomes de porc dans les 14 sous-clones étudiés est donnée tableau 1. Les 7 sous-clones positifs pour la synténie ont retenu le chromosome 3 dans 90 p. 100 des mitoses analysées. Pour 6 sous-clones négatifs, le chromosome 3 est absent dans toutes les mitoses. Un clone, PFA10, est faiblement discordant : négatif pour la synténie, le chromosome 3 est présent dans 33 p. 100 des mitoses analysées. Aucune autre corrélation n'a pu être établie avec cette série de sous-clones. 


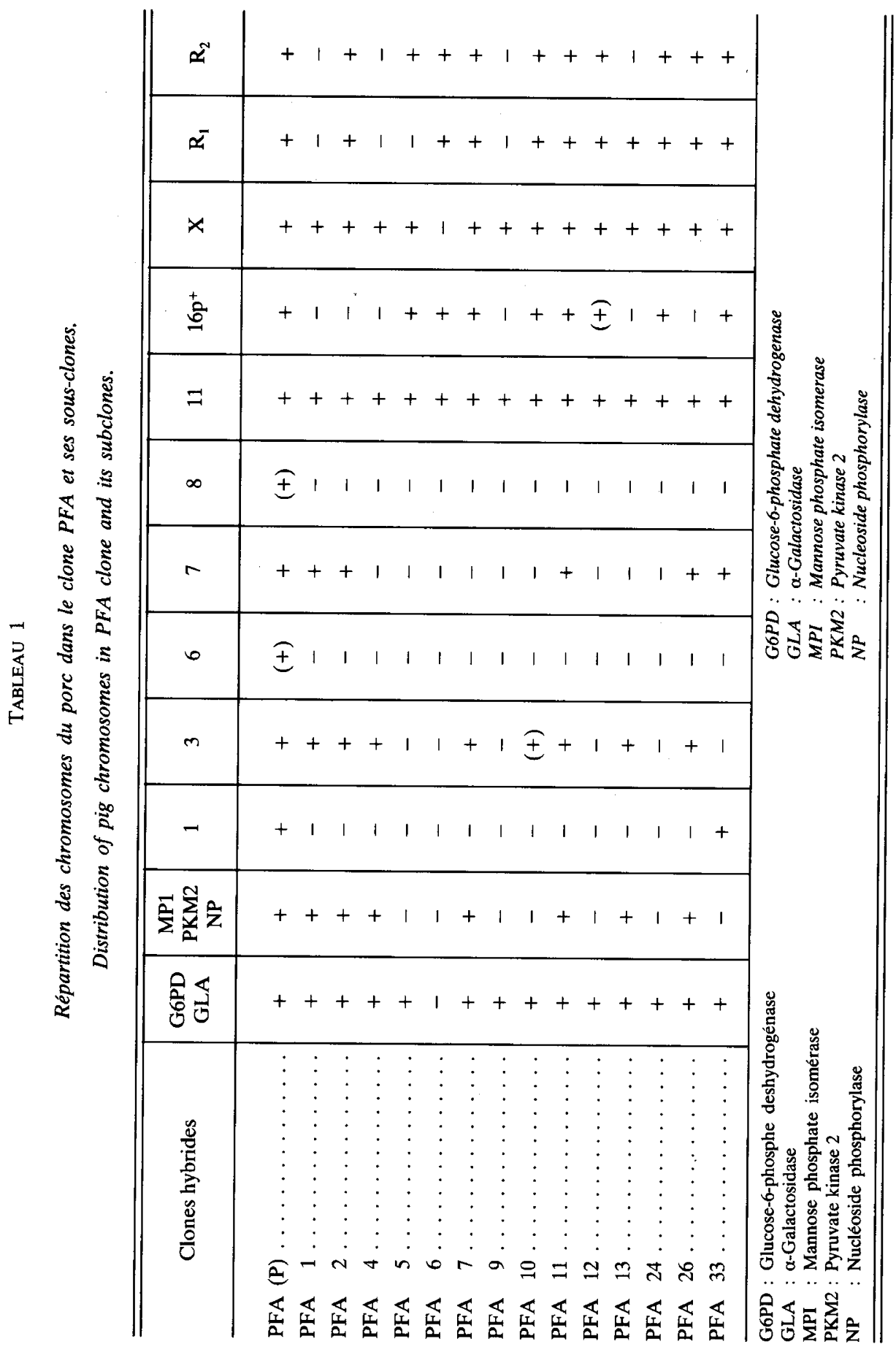




\section{Discussion}

La lignée wg3h est souvent utilisée comme souche cellulaire parentale dans la fabrication de clones hybrides à partir de cellules d'animaux domestiques car elle donne un bon pourcentage de fusion, contrairement à d'autres souches de hamster chinois comme CHO. La connaissance des remaniements qui la caractérise permet alors de faciliter l'identification des chromosomes d'un clone hybride. Isolée depuis 1971 à partir de la lignée DON, elle a subi son évolution cytogénétique propre et les chromosomes perdus ou remaniés qu'elle comporte sont différents de ceux des autres souches permanentes de hamster chinois aussi anciennes et dont le caryotype en bandes $\mathbf{G}$ a été étudié en détail : CHO (WORTON et al., 1977), V79 (ThACKER, 1981).

La présence d'un clone discordant, PFA10, sur 14 étudiés donne un taux de discordance de 7 p. 100 , ce qui est inférieur au taux de 15 p. 100 généralement admis (VAN Cong, 1978). Ce clone est discordant dans le sens ch. $3^{+}$, synténie ${ }^{-}$, mais cette discordance est faible, car le chromosome 3 n'est rencontré que dans 33 p. 100 des mitoses. Ce chiffre est à la limite de ce qui est accepté pour admettre qu'un chromosome est présent dans un hybride (VAN CONG, 1978). Des exemples de localisation avec 1 ou 2 discordants dans le sens $\mathrm{ch}^{+}{ }^{+}$, synténie ${ }^{-}$sont fréquents en cartographie humaine. Ils ne sont jamais clairement interprétés mais généralement les localisations ont été confirmées par la suite : en exemple, la localisation de ADK (Adenosine kinase) sur le chromosome 10 humain (KLOBUTCHER et al., 1976). Une interprétation possible pourrait être l'inactivation de gènes de ce chromosome dans le clone considéré. Une semblable inactivation existe pour des autosomes (GodBout et al., 1983).

La répartition des enzymes MPI, PKM2, NP chez les mammifères étudiés a déjà été en partie décrite (GELlin et al., 1981 ; O'BRIEN \& NASH, 1982). Trois situations sont observées :

A. MPI, PKM2 sont synténiques entre eux mais non avec NP. Deux chromosomes acrocentriques sont alors porteurs (les chromosomes des espèces sont nommés par un chiffre, précédé d'un code de 3 lettres en accord avec « An International System for Human Cytogenetic Nomenclature », 1978). Cette situation se rencontre dans les espèces suivantes :

- l'homme (Homo sapiens), MPI, PKM2 : chromosome HSA15 (VAN HEYNINGEN et al., 1974), NP : HSA14 (CREAGAN et al., 1973) ;

- le chimpanzé (Pan troglodytes), MPI, PKM2 : PTR16. NP : PTR15 (PEARson et al., 1977) ;

- le gorille (Gorilla gorilla), MPI, PKM2 : GGO15. NP : GGO18 (PEARsON et al., 1977) ;

- la souris (Mus musculus), MPI, PKM2 : MMU9. NP : MMU14 (DAvisson \& RoDERICK, 1982).

B. PKM2, NP sont synténiques entre eux mais non avec MPI. C'est le cas du mouton (Ovis aries). Les chromosomes porteurs ne sont pas connus (SAIDI-MEHTAR \& HoRs-CAYLA, 1981).

C. MPI, PKM2, NP sont synténiques. Cette situation est rencontrée dans les espèces suivantes :

- le lémurien Microcebus (Microcebus murinus), chromosome MIM2 (COCHET et al., 1982) ; 
- le macaque (Macaca mulatta), chromosome MML7 (EsTOP et al., 1983 ; RoDERICK et al., 1984);

- le singe Aotus (Aotus aotus), chromosome AAO11 (MA, 1983) ;

- le chat (Felix cattus), chromosome FCAB3 (O'BRIEN \& NASH, 1982) ;

- le porc (Sus scrofa), chromosome SSC3.

Chez le chat et le porc, ces 3 gènes sont portés par un chromosome submétacentrique, présentant au niveau des bras longs, 3 bandes $G$ distinctes, la bande médiane étant la plus intense. La connaissance fine du chromosome SSC3 est encore insuffisante pour pouvoir détailler une homologie entre le chat et le porc comme celle décrite entre le chat et l'homme (NASH \& O'BRIEN, 1982), le chat et les primates (DuTRILlauX \& Couturier, 1983), le lapin et l'homme (Dutrillaux et al., 1980). Chez le macaque, les bras longs du chromosome MML7 présentent une répartition des bandes analogue à celle du chromosome acrocentrique HSA14 (PEARSON et al., 1978). D'après EsToP et al. (1983), le chromosome MML7 résulterait d'une fusion centrique suivi d'une inversion péricentrique ou d'un remaniement terminal. La distribution des gènes MPI, PKM2, NP sur 2 chromosomes comme chez l'homme est alors ancestrale. DUTRILLAUX \& COUTURIER (1983) font une comparaison entre un caryotype ancestral carnivore et un caryotype ancestral primate. Dans les 2 cas, un seul chromosome représente l'équivalent de HSA14 et HSA15. Ils déduisent de cette comparaison que le chromosome du chat FCAB3 a gardé une intégrité ancestrale. La scission en 2 chromosomes comme chez l'homme s'interprète alors comme un phénomène plus récent.

Reçu le 18 août 1983.

Accepté le 20 février 1984.

\section{Références bibliographiques}

ANDResen E., BaKer L.N., 1964. The $\mathrm{C}$ blood group system in pigs and the detection and estimation of linkage between the $\mathrm{C}$ and $\mathrm{J}$ systems. Genetics, 49, 379-386.

Cochet C., Creau-Goldberg N., Turleau C., Grouchy J. de, 1982. Gene mapping of Microcebus murinus (Lemuridae). A comparison with man and Cebus capucinus (Cebidae). Cytogenet. Cell Genet., 33, 213-221.

Creagan R.P., Tan Y.H., Chen S., Tischfield J.A., Ruddle F.H., 1974. Mouse/human somatic cell hybrids utilizing human parental cells containing a (14/22) translocation : assignment of the gene for nucleoside phosphorylase to chromosome 14. Proceedings of the first International Conference on Human Gene Mapping, New Haven, June 10-13, 1973. Cytogenet. Cell Genet., 13, 83-85.

Davisson M.T., Roderick T.H., 1982. The linkage map of the mouse. In : O'BriEn S.J. (ed.), Genetic Maps, 2, 277-286, Frederick U.S.A.

DutrillauX B., Couturier J., 1983. The ancestral karyotype of Carnivora comparison with that of platyrrhine monkeys. Cytogenet. Cell Genet., 35, 200-208.

Dutrillaux B., Viegas-Pequignot E., Couturier J., 1980. Très grande analogie de marquage chromosomique entre le lapin (Oryctolagus cuniculus) et les primates, dont l'homme. Ann. Génét., 23, 22-25.

ECHARD G., 1974. Chromosomal banding patterns and karyotype evolution in three pig kidney cell strains (PK15, F and RP). Chromosoma, 45, 133-149. 
Estop A.M., Garver J.J., Egozcue J., Meera Khan P., Pearson P.L., 1983. Complex chromosome homologies between the rhesus monkey (Macaca mulatta) and man. Cytogenet. Cell Genet., 35, 46-50.

Fórster M., Stranzinger G., Hellkuhl B., 1980. X-chromosome gene assignment of swine and cattle. Naturwissenschaften, 67, 48.

Francke U., Oliver N., 1978. Quantitative analysis of high-resolution trypsin-giemsa bands on human prometaphase chromosomes. Human Genet., 45, 137-165.

Gellin J., Benne F., Renard C., Vaiman M., Hors-Cayla M.C., Gillois M., 1979. Pig gene mapping : synteny, attempt to assign the histocompatibility complex (SLA). Proceedings of the Vth International Conference on Human Gene Mapping, Edinburgh, July 9-13 1979. Cytogenet. Cell Genet., 25, 159.

Gellin J., Benne F., Hors-Cayla M.C., Gillois M., 1980. Carte génique du porc (Sus scrofa L.). I. Etude de deux groupes synténiques G6PD, PGK, HPRT et PKM2, MPI. Ann. Génét., 23, $15-21$.

Gellin J., Echard G., Benne F., Gillois M., 1981. Pig gene mapping : PKM2-MPI-NP synteny. Cytogenet. Cell. Genet., 30, 59-62.

Godbout R., Dryja T.P., Souire J., Gallie B.L., Phillips R.A., 1983. Somatic inactivation of genes on chromosome 13 is a common event in retinoblastoma. Nature, 304, 451-453.

Hruban V., Simon M., HRAdecky J., JiLek F., 1976. Linkage of the pig main histocompatibility complex and the $\mathrm{J}$ blood group system. Tissue Antigens, 7, 267-271.

I.S.C.N., 1978. An International System for Human Cytogenetic Nomenclature. Cytogenet. Cell Genet., 21, 309-404.

I.S.G.N., 1979. International System for Human Gene Nomenclature. Cytogenet. Cell Genet., 25, 96-116.

Klobutcher L.A., Nichols E.A., Kucherlapati R.S., Ruddle F.H., 1976. Assignment of the gene for human adenosine kinase to chromosome 10 using a somatic cell hybrid clona panel. Proceedings of the IIIrd International Conference on Human Gene Mapping, Baltimore, October 5-7, 1975. Cytogenet. Cell Genet., 16, 171-174.

LEONG M.M., LIN C.C., RUTH R.F., 1982. The localization of HPRT, G6PD and GLA on the $\mathrm{X}$-chromosome of the domestic pig. Proceedings of the VIth International Conference on Human Gene Mapping, Oslo, June 29 to July 3, 1981. Cytogenet. Cell Genet., 32, 295.

MA N.S.F., 1983. Comparative gene mapping of the owl monkey, Aotus, with karyotypes V $(2 \mathrm{n}=46)$ and VI $(2 \mathrm{n}=49.50)$. Cytogenet. Cell Genet., 35, 117-123.

NASH W.G., O'BRIEN S.J., 1982. Conserved regions of homologous G-banded chromosomes between orders in mammalian evolution: Carnivores and primates. Proc. Natl. Acad. Sci. USA, 79, 6631-6635.

O'BRIEN S.J., NASH W.G., 1982. Genetic mapping in mammals : chromosome map of domestic cat. Science, 216, 257-265.

Ollivier L., Sellier P., 1982. Pig genetics : a review. Ann. Génét. Sél. Anim., 14, 481-544.

Pearson P.L., Garver J.J., Estop A., Dijksman T.M., Wisnen L.M.M., Meera Khan P., 1978. Gene assignments to the presumptive homologs of human chromosomes $2,9,13,14$ and 15 in the Pongidae and Cercopithecoidea. Proceedings of the IVth International Conference on Human Gene Mapping, Winnipeg, August 14-18, 1977. Cytogenet. Cell Genet., 22, 588-593.

Proceedings of the first International Conference for the Standardization of Banded Karyotypes of Domestic Animals (Reading 1976), 1980. Hereditas, 92, 145-162.

RaY M., Mohandas T., 1976. Proposed banding nomenclature for the chinese hamster chromosomes (Cricetulus griseus). Proceedings of the IIIrd International Conference on Human Gene Mapping, Baltimore, October 5-7, 1975. Cytogenet. Cell Genet., 16, 83-91.

Roderick T.H., Lalley P.A., Davisson M.T., O'Brien S.J., Womack J.E., Creau-Goldberg N., ECHARD G., MOORE K.L., 1984. Report of the committee on comparative mapping. Proceedings of the VIIth International Conference on Human Gene Mapping, Los Angeles, August 21-26, 1983. Cytogenet. Cell Genet., 37, 312-339.

Saidi-Mehtar N., Hors-CaYla M.C., 1981. Sheep gene mapping by somatic cell hybridization. III. Synteny between pyruvate kinase M2 (PKM2) and nucleoside phosphorylase (NP) in domestic sheep. Ann. Génét., 24, 148-151. 
THACKER J., 1981. The chromosome of a V79 chinese hamster line and a mutant subline lacking HPRT activity. Cytogenet. Cell Genet., 29, 16-25.

VAN CONG N., 1978. Cartographie chromosomique humaine par la méthode d'hybridation cellulaire homme-rongeur et application à l'étude génétique des maladies héréditaires du métabolisme. Thèse de Doctorat d'Etat. Université Paris VI.

Van Heyningen V., Bobrow M., Bodmer W.F., Povey S., Gardiner S.E., Hopkinson D.A., 1975. Assignment of the genes for mitochondrial malate dehydrogenase to chromosome 7 , for mannose phosphate isomerase and pyruvate kinase to chromosome 15 , and, probably, for human esterase-D to chromosome 13 using man-house hybrids. Proceedings of the IInd International Conference on Human Gene Mapping, Rotterdam, July 7-10, 1974. Cytogenet. Cell Genet., 14, 353-357.

WANG H.C., FEDOROFF S., 1972. Banding in human chromosomes treated with trypsin. Nat. New Biol., 235, 52-54.

Westerveld A., Visser R.P.L.S., Meera Khan P., Bootsma D., 1971. Loss of human genetic markers in man-chinese hamster somatic cell hybrids. Nat. New Biol., 234, 20-24.

Worton R.G., Ho C.C., Duff C., 1977. Chromosome stability in CHO cells. Somat. Cell Genet., 3, 27-45. 